\title{
ASYMPTOTICS OF THE NUMBER OF $k$-WORDS WITH AN $\ell$-DESCENT
}

\author{
Amitai Regev* \\ Department of Mathematics \\ The Pennsylvania State University \\ University Park, PA 16802, U.S.A \\ E-mail: regev@math.psu.edu \\ and \\ Department of Theoretical Mathematics \\ The Weizmann Institute of Science \\ Rehovot 76100, Israel \\ E-mail: regev@wisdom.weizmann.ac.il
}

Submitted: December , 1997; Accepted: February 25, 1998

\begin{abstract}
The number of words $w=w_{1} \cdots w_{n}, 1 \leq w_{i} \leq k$, for which there are $1 \leq i_{1}<\cdots<i_{\ell} \leq n$ and $w_{i_{1}}>\cdots>w_{i_{\ell}}$, is given, by the Schensted-Knuth correspondence, in terms of standard and semi-standard Young tableaux. When $n \rightarrow \infty$, the asymptotics of the number of such words is calculated.
\end{abstract}

*Work partially supported by N.S.F. Grant No. DMS-94-01197. 


\section{The Main Results.}

Let $k, n>0$ be integers and let $W(k ; n)=\left\{w_{1} \cdots w_{n} \mid 1 \leq w_{i} \leq k\right.$ for all $\left.1 \leq i \leq n\right\}$ denote the set of words of length $n$ on the alphabet $\{1, \cdots, k\}$. A word $w=w_{1} \cdots w_{n} \in$ $W(k, n)$ is said to have a descent of length $\ell$ if there exist indices $1 \leq i_{1}<\cdots<i_{\ell} \leq n$ such that $w_{i_{1}}>\cdots>w_{i_{\ell}}$ (trivially, such words exist if and only if $\ell \leq k$ ).

Let $W(k, \ell ; n)$ denote the set of words in $W(k ; n)$ having descent $\leq \ell$, and denote $w(k, \ell ; n)=|W(k, \ell ; n)|$. Thus $W(k ; n)=W(k, k ; n)$, and $w(k, k ; n)=k^{n}$.

Recall: given two sequences $\left\{a_{n}\right\}$ and $\left\{b_{n}\right\}$ of real numbers, we denote $a_{n} \underset{n \rightarrow \infty}{\stackrel{\simeq}{\rightarrow}} b_{n}$ (or simply $\left.a_{n} \simeq b_{n}\right)$ if $\lim _{n \rightarrow \infty} \frac{a_{n}}{b_{n}}=1$.

The main result here is

Theorem 1. Let $1 \leq \ell \leq k$, then

$$
w(k, \ell ; n) \underset{n \rightarrow \infty}{\simeq} \frac{1 ! 2 ! \cdots(\ell-1) !}{(k-\ell) ! \cdots(k-1) !} \cdot\left(\frac{1}{\ell}\right)^{\ell(k-\ell)} \cdot n^{\ell(k-\ell)} \cdot \ell^{n}
$$

Remark. $\frac{1 ! \cdots(\ell-1) !}{(k-\ell) ! \cdots(k-1) !}=\left[\frac{k ! !}{\ell ! !(k-\ell) ! !}\right]^{-1}$, where $m ! ! \stackrel{\text { def }}{=} 1 ! 2 ! \cdots(m-1) !$

Standard and Semistandard Tableaux.

Let $\lambda \vdash n$ (i.e. $\lambda$ is a partition of $n$ ). A tableau of shape $\lambda$, filled with $1, \cdots, n$, is standard if the numbers in it are increasing both in rows and in columns. Let $d_{\lambda}$ denote the number of such tableaux. It is well known that $d_{\lambda}=\operatorname{deg}\left(\chi_{\lambda}\right)$, where $\chi_{\lambda}$ is the corresponding irreducible character of the symmetric group $S_{n}$.

A $k$-tableau of shape $\lambda$ is a tableau filled with $1, \cdots, k$ possibly with repetitions; it is semi-standard if the numbers are weakly increasing in rows and strictly increasing in columns. Let $s_{k}(\lambda)$ denote the number of such $k$-tableaux. It is well known that $s_{k}(\lambda)$ is the degree of a corresponding irreducible character of $G L(k, \mathbb{C})$ (or of $S L(k, \mathbb{C})$ ).

The numbers $w(k, \ell ; n)$ are given by

Theorem 2. Let $\wedge_{\ell}(n)=\left\{\left(\lambda_{1}, \lambda_{2}, \cdots\right) \vdash n \mid \lambda_{\ell+1}=0\right\}$. Then

$$
w(k, \ell ; n)=\sum_{\lambda \in \wedge_{\ell}(n)} s_{k}(\lambda) \cdot d_{\lambda}
$$

Formulas for calculating $d_{\lambda}$ 's and $s_{k}(\lambda)$ 's are well known. Here we shall need the following formula:

Let $\lambda=\left(\lambda_{1}, \lambda_{2}, \cdots\right)$. If $\lambda_{k+1}>0$ then $s_{k}(\lambda)=0$. Assume $\lambda_{k+1}=0$. Then

$$
s_{k}(\lambda)=[1 ! 2 ! \cdots(k-1) !]^{-1} \cdot \prod_{1 \leq i<j \leq k}\left(\lambda_{i}-\lambda_{j}+j-i\right)
$$


We turn now to the proofs of Theorems 1 and 2, starting with

\section{The proof of Theorem 2 :}

Apply the Schensted-Knuth correspondence $[\mathrm{K}]$ to $w \in W(k ; n): w \rightarrow\left(P_{\lambda}, Q_{\lambda}\right)$, where $P_{\lambda}$ and $Q_{\lambda}$ are tableaux of same shape $\lambda, Q_{\lambda}$ is standard and $P_{\lambda}$ is $k$-semistandard. This gives a bijection

$$
W(k ; n) \leftrightarrow\left\{\left(P_{\lambda}, Q_{\lambda}\right) \mid \lambda \in \wedge_{k}(n), P_{\lambda} \text { is } k \text {-semistandard, } Q_{\lambda} \text { is standard }\right\}
$$

Moreover, let $w \leftrightarrow\left(P_{\lambda}, Q_{\lambda}\right)$ under this correspondence, then $w$ has a descent of length $\geq r$ if and only if $\lambda_{r} \supsetneqq 0$. It clearly follows that the Schensted-Knuth correspondence gives a bijection

$$
W(k, \ell ; n) \leftrightarrow\left\{\left(P_{\lambda}, Q_{\lambda}\right) \mid \lambda \in \wedge_{\ell}(n), P_{\lambda} \text { is } k \text {-semistandard, } Q_{\lambda} \text { is standard }\right\}
$$

Hence

$$
w(k, \ell ; n)=\sum_{\lambda \in \Lambda_{\ell}(n)} s_{k}(\lambda) d_{\lambda}
$$

Q.E.D.

Remark. Let $1 \leq \ell \leq k$ and let $\lambda \in \wedge_{\ell}(n)$, then it is easy to verify that $(*)$ implies that

$$
s_{k}(\lambda)=a \cdot b \cdot c
$$

where $a=[(k-\ell) ! \cdots(k-1) !]^{-1}, b=\prod_{1 \leq i \leq \ell}\left[\prod_{\ell+1 \leq j \leq k}\left(\lambda_{i}+j-i\right)\right]$ and $c=\prod_{1 \leq i<j \leq \ell}\left(\lambda_{i}-\lambda_{j}+j-i\right)$

\section{The Proof of Theorem 1.}

Here the results of $[\mathrm{C} . \mathrm{R}]$ are applied. Let $\lambda \in \wedge_{\ell}(n), 1 \leq \ell \leq k$, and write:

$$
\lambda=\left(\lambda_{1}, \cdots, \lambda_{\ell}\right)=\left(\lambda_{1}, \cdots, \lambda_{k}\right) \text {, where } \lambda_{\ell+1}=\cdots=\lambda_{k}=0
$$

Also write $\lambda_{j}=\frac{n}{\ell}+c_{j} \sqrt{n}$. By the notations of [C.R], the factors $b$ and $c$ of $(* *)$ satisfy

$$
b \approx \prod_{1 \leq i \leq \ell}\left(\frac{n}{\ell}\right)^{k-\ell}=\left(\frac{n}{\ell}\right)^{\ell(k-\ell)}
$$

and

$$
c \approx\left[\prod_{1 \leq i<j \leq \ell}\left(c_{i}-c_{j}\right)\right](\sqrt{n})^{\frac{\ell(\ell-1)}{2}}
$$


Thus

$$
s_{k}(\lambda) \approx\left[(k-\ell) ! \cdots(k-1) ! \ell^{\ell(k-\ell)}\right]^{-1} \cdot\left[\prod_{1 \leq i<j \leq \ell}\left(c_{i}-c_{j}\right)\right] \cdot n^{\ell(k-\ell)+\frac{\ell(\ell-1)}{4}} .
$$

Apply now [C.R. Theorem 2] with $\beta=1$ (also $\ell$ replacing $k$ and $s_{k}(\lambda)$ replacing $f(\lambda)$ ):

$$
\begin{aligned}
& w(k, \ell ; n) \underset{\operatorname{Thm} 1}{=} \sum_{\lambda \in \wedge_{\ell}(n)} s_{k}(\lambda) d_{\lambda} \simeq \\
& \simeq\left[(k-\ell) ! \cdots(k-1) ! \cdot \ell^{\ell(k-\ell)}\right]^{-1} \cdot\left(\frac{1}{\sqrt{2 \pi}}\right)^{\ell-1} \cdot \ell^{\frac{1}{2} \ell^{2}} \cdot n^{\ell(k-\ell)} \cdot \ell^{n} \cdot I_{\ell},
\end{aligned}
$$

where

$$
I_{\ell}=\int \cdots \int_{\substack{x_{1}+\cdots+x_{\ell}=0 \\ x_{1} \geq \cdots \geq x_{\ell}}}\left[\prod_{1 \leq i<j \leq \ell}\left(x_{i}-x_{j}\right)\right]^{2} \exp \left(-\frac{\ell}{2} \sum_{j=1}^{\ell} x_{j}^{2}\right) d^{(\ell-1)} x .
$$

Special Case: Let $\ell=k$. Then $w(k, k ; n)=k^{n}$. Cancelling $k^{n}$ from both sides of $(* * *)$ implies that

$$
I_{k}=[1 ! 2 ! \cdots(k-1) !] \sqrt{2 \pi}^{k-1} \cdot\left(\frac{1}{k}\right)^{\frac{1}{2} k^{2}}
$$

(Note: by $[\mathrm{R}, \S 4] I_{k}$ can also be calculated by the Mehta-Selberg integral).

In particular,

$$
I_{\ell}=[1 ! 2 ! \cdots(\ell-1) !] \sqrt{2 \pi}^{\ell-1} \cdot\left(\frac{1}{\ell}\right)^{\frac{1}{2} \ell^{2}} .
$$

Substituting for $I_{\ell}$ in $(* * *)$ implies that

$$
w(k, \ell ; n) \simeq \frac{1 ! 2 ! \cdots(\ell-1) !}{(k-\ell) ! \cdots(k-1) !} \cdot\left(\frac{1}{\ell}\right)^{\ell(k-\ell)} \cdot n^{\ell(k-\ell)} \cdot \ell^{n}
$$

which completes the proof of Theorem 1.

Q.E.D.

Acknowledgement. I am thankful to Dr. H. Wilf for suggesting this problem.

\section{REFERENCES}

[C.R] P. S. Cohen, A. Regev, Asymptotics of combinatorial sums and the central limit theorem, SIAM J. Math. Anal., Vol 19, No 5 (1980) 1204-1215.

[K] D. E. Knuth, The Art of Computer Programming, Vol. 3, Addison-Wesley, Reading, Mass., 1968.

[R] A. Regev, Asymptotic values for degrees associated with strips of Young diagrams, Adv. in Math. 41 (1981), 115-136. 